\title{
The spherical nanoparticle-encapsulated chlorhexidine enhances anti-biofilm efficiency through an effective releasing mode and close microbial interactions
}

\author{
This article was published in the following Dove Press journal: \\ International Journal of Nanomedicine \\ 31 May 2016 \\ Number of times this article has been viewed
}

\author{
Xuan $\mathrm{Li}^{1}$ \\ Chi-Hin Wong ${ }^{2}$ \\ Tsz-Wing $\mathrm{Ng}^{2}$ \\ Cheng-Fei Zhang' \\ Ken Cham-Fai Leung ${ }^{2}$ \\ Lijian Jin' \\ 'Faculty of Dentistry, The University \\ of Hong Kong, ${ }^{2}$ Department of \\ Chemistry, Institute of Creativity \\ and Partner State Key Laboratory of \\ Environmental and Biological Analysis, \\ Hong Kong Baptist University, Hong \\ Kong SAR, People's Republic of China
}

Correspondence: Lijian Jin

Faculty of Dentistry, The University of Hong Kong, 34 Hospital Road, Hong

Kong SAR, People's Republic of China

$\mathrm{Tel}+85228590302$

Fax +852 28587874

Email ljjin@hku.hk

Ken Cham-Fai Leung

Department of Chemistry, Institute

of Creativity and Partner State Key

Laboratory of Environmental and

Biological Analysis, Hong Kong Baptist

University, Kowloon Tong, Hong Kong

SAR, People's Republic of China

$\mathrm{Tel}+852341 \mid 2319$

Fax+852 34II 7348

Email cfleung@hkbu.edu.hk

\begin{abstract}
We reported two forms (sphere and wire) of newly fabricated chlorhexidine (CHX)-loaded mesoporous silica nanoparticles (MSNs), and investigated their releasing capacities and anti-biofilm efficiencies. The interactions of the blank MSNs with planktonic oral microorganisms were assessed by field emission scanning electron microscopy. The anti-biofilm effects of the two forms of nanoparticle-encapsulated CHX were examined by 2,3-bis (2-methoxy4-nitro-5-sulfo-phenyl)-2H-tetrazolium-5-carboxanilide. The profiles of biofilm penetration were analyzed by fluorescent-labeled MSNs using confocal microscopy and ImageJ. The spherical MSNs with an average diameter of $265 \mathrm{~nm}$ exhibited a larger surface area and faster CHX-releasing rate than the MSN wires. The field emission scanning electron microscopy images showed that both shaped MSNs enabled to attach and further fuse with the surfaces of testing microbes. Meanwhile, the nanoparticle-encapsulated CHX could enhance the anti-biofilm efficiency with reference to its free form. Notably, the spherical nanoparticle-encapsulated CHX presented with a greater antibiofilm capacity than the wire nanoparticle-encapsulated CHX, partly due to their difference in physical property. Furthermore, the relatively even distribution and homogeneous dispersion of spherical MSNs observed in confocal images may account for the enhanced penetration of spherical nanoparticle-encapsulated CHX into the microbial biofilms and resultant anti-biofilm effects. These findings reveal that the spherical nanoparticle-encapsulated CHX could preferably enhance its antibiofilm efficiency through an effective releasing mode and close interactions with microbes.
\end{abstract}

Keywords: mesoporous silica nanoparticles, morphology, chlorhexidine, imaging, biofilms

\section{Introduction}

In recent years, nanotechnology has been increasingly used in the development of a broad range of biomedical agents and approaches. Among different types of inorganic nanomaterials, the mesoporous silica nanoparticles (MSNs) exhibit many unique features, such as large surface area, high pore volume, stable physicochemical property, and flexible surface modification, which make MSN a potential drug delivery vehicle. ${ }^{1}$ Previous in vitro and in vivo studies have reported the promising capacity of MSNs as anticancer and antibacterial drug delivery systems. ${ }^{2-6}$ Moreover, our group has recently successfully encapsulated the most commonly used antimicrobial agent in dentistry, chlorhexidine (CHX), into commercial MSNs (MCM-41), and further confirmed their antibacterial and anti-biofilm efficiencies via an effective releasing mode. ${ }^{7}$

There are plenty of studies on how the nanoparticle morphology, size, and surface modifications affect their interactions with the mammal cells. ${ }^{8,9}$ Currently, there are 
limited reports on the interactions of MSNs with the microbes and especially the possible effects of nanoparticle morphology on microbial activities. Hence, we herewith reported the physical characterization of two representative morphologies (sphere and wire) of newly fabricated CHX-loaded MSNs, and evaluated their releasing capacities and anti-biofilm efficiencies. Additionally, the fluorescent MSNs were prepared as a tracking tool to further investigate the diffusion profiles of the MSNs into the microbial biofilms.

\section{Materials and methods Chemicals}

Cetyltrimethylammonium bromide (CTAB), 3-aminopropyltriethoxysilane (APTES), rhodamine B isothiocyanate (RITC), and CHX were purchased from Sigma-Aldrich Co. (St Louis, MO, USA). Tetraethyl orthosilicate (TEOS) was purchased from Acros Organics (Thermo Fisher Scientific, Waltham, MA, USA). Aqueous ammonia was purchased from VWR (Radnor, PA, USA). Hydrochloric acid (37\%) was purchased from RCI Labscan (Bangkok, Thailand).

\section{Microorganisms}

The oral microorganisms Streptococcus sobrinus (ATCC 33478), Streptococcus mutans (ATCC 35668), and Candida albicans (SC5314) were obtained from the archival collection of Department of Oral Biosciences, Faculty of Dentistry, University of Hong Kong. S. sobrinus and S. mutans were inoculated on horse blood agar plates (Oxoid, Thermo Fisher Scientific) with 5\% horse blood (Hemostat Laboratories, Dixon, CA, USA) and incubated in anaerobic chamber with $5 \% \mathrm{CO}_{2}, 10 \% \mathrm{H}_{2}$, and $85 \% \mathrm{~N}_{2}$ at $37^{\circ} \mathrm{C}$. C. albicans were cultured on Sabouraud dextrose agar (Thermo Fisher Scientific) and incubated aerobically at $37^{\circ} \mathrm{C}$. Before the assays, the microorganisms were suspended in brain heart infusion (BHI) broth (Oxoid) (for bacteria) or yeast nitrogen base (YNB) broth (Difco, Franklin Lakes, NJ, USA) containing $50 \mathrm{mM}$ glucose (for C. albicans) overnight before using.

\section{Synthesis and characterization of MSNs}

Different shaped MSNs were prepared by adjusting the CTAB concentration in ammonia-catalyzed TEOS hydrolysis reaction. Briefly, a certain amount of $\mathrm{CTAB}$ was dissolved completely in the water at $30^{\circ} \mathrm{C}$ to get the clear solution. After adding the ammonia, TEOS was mixed with $10 \mathrm{~mL}$ ethanol and then dropped at a rate of $1 \mathrm{~mL} / \mathrm{min}$ with vigorous stirring ( $250 \mathrm{rpm} / \mathrm{min}$ ) for 5 hours at room temperature. The spherical MSNs (S-MSNs) were synthesized as described by Yu et $\mathrm{al}^{10}$ with some modifications, and the weight or volume of the reactants was $0.364 \mathrm{~g}(\mathrm{CTAB}): 180 \mathrm{~mL}\left(\mathrm{H}_{2} \mathrm{O}\right): 5 \mathrm{~mL}$ $\left(\mathrm{NH}_{3} \cdot \mathrm{H}_{2} \mathrm{O}\right): 1.56 \mathrm{~mL}$ (TEOS). For the $\mathrm{MSN}$ wires (W-MSNs), the reactant amount was $0.8 \mathrm{~g}$ (CTAB): $200 \mathrm{~mL}\left(\mathrm{H}_{2} \mathrm{O}\right)$ : $3.5 \mathrm{~mL}\left(\mathrm{NH}_{3} \cdot \mathrm{H}_{2} \mathrm{O}\right): 1 \mathrm{~mL}(\mathrm{TEOS})$. The well-synthesized MSNs were obtained by centrifugation at $6,000 \mathrm{rpm}$ for 10 minutes using centrifuge 5804 (Eppendorf, Hamburg, Germany). The surfactant was removed in acidic ethanol by sonication (20 minutes) twice, and the residue was confirmed by infrared spectrometry. The particle morphology and pore structure were observed under LEO1530 field emission scanning electron microscopy (FE-SEM; Carl Zeiss Meditec AG, Jena, Germany) and Tecnai G2 20 S-TWIN transmission electron microscopy (FEI, Hillsboro, OR, USA). The nanoparticle surface areas and pore size were analyzed by $\mathrm{N}_{2}$ adsorption-desorption isotherms at about $77 \mathrm{~K}$ using ASAP 2020M system (Micromeritics, Norcross, GA, USA).

\section{$\mathrm{CHX}$ loading and releasing}

The CHX solution ( $2 \mathrm{mg} / \mathrm{mL}$ ) was freshly prepared by dissolving $0.02 \mathrm{~g}$ of CHX in $10 \mathrm{~mL}$ of hot ethanol. In all, $0.1 \mathrm{~g}$ of well-synthesized MSNs was subsequently immersed in the $\mathrm{CHX}$ solution with physical stirring at room temperature for 24 hours. We harvested nanoparticle-encapsulated CHX (Nano-CHX) by centrifugation at 6,000 rpm for 10 minutes and washed them with water and ethanol, once each. The final products were obtained after oven drying at $90^{\circ} \mathrm{C}$ and denoted as S-CHX and W-CHX for spherical nanoparticleencapsulated $\mathrm{CHX}$ and wire nanoparticle-encapsulated $\mathrm{CHX}$, respectively. The loading efficiencies of CHX in S-/W-CHX were determined by TGA-6 Thermogravimetric Analyzer (TGA; PerkinElmer Inc., Waltham, MA, USA). Both the loading process and TGA determination were repeated for three times to compare the loading capacity of two different shaped MSNs. Referring to the releasing profiles of S- and W-CHX, $10 \mathrm{mg}$ of both shaped Nano-CHX was dispersed in $5 \mathrm{~mL}$ of distilled water and then shaken at $37^{\circ} \mathrm{C}$. At each time point, the releasing system was centrifuged to collect the supernatant, and subsequently re-suspended in $5 \mathrm{~mL}$ of distilled water again. The absorbance of the supernatant was measured at $254 \mathrm{~nm}$ by Cary UV-100 UV/visible absorption spectroscopy (Agilent Technologies, Santa Clara, CA, USA).

\section{Surface modification and fluorescent labeling of MSNs}

MSNs $(0.5 \mathrm{~g})$ were dispersed in $100 \mathrm{~mL}$ of ethanol and refluxed with $1.25 \mathrm{~mL}$ of APTES overnight in nitrogen. The amine-modified MSNs were centrifuged and washed with ethanol twice to remove the unreacted APTES. Then, 
the fluorescent MSNs were prepared by a reaction between RITC and the amine groups on the modified MSNs. ${ }^{11}$ The air-dried amine-MSNs $(0.2 \mathrm{~g})$ were dispersed in $40 \mathrm{~mL}$ of ethanol containing $5 \mathrm{mg}$ of RITC with vigorous stirring for 4 hours at room temperature. The fluorescent-labeled MSNs were washed with ethanol for three times and collected by centrifugation at 6,000 rpm for 5 minutes (Eppendorf). The vacuum-dried final products were denoted as RITC-SMSNs and RITC-W-MSNs, respectively. The fluorescent intensities of RITC-S-/RITC-W-MSNs $(100 \mu \mathrm{g} / \mathrm{mL})$ and RITC $(28 \mu \mathrm{g} / \mathrm{mL})$ in ethanol were determined by LS 50B fluorescence spectrometer (PerkinElmer Inc.).

\section{Field emission scanning microscopy}

Briefly, $0.5 \mathrm{~mL}$ of bacteria $\left(10^{6}\right.$ cells $\left./ \mathrm{mL}\right)$ or yeast $\left(10^{3}\right.$ cells $\left./ \mathrm{mL}\right)$ suspension and $0.5 \mathrm{~mL}$ of $\mathrm{S}-/ \mathrm{W}-\mathrm{MSNs}$ dispersion $(200 \mu \mathrm{g} / \mathrm{mL})$ were mixed in the sterilized plastic tubes and incubated at $37^{\circ} \mathrm{C}$ for 24 hours. Then, the mixture was processed by vortexing (Stuart Auto Vortex Mixer SA2, Rhys International Ltd, Greater Manchester, UK) for 3 seconds. In all, $10 \mu \mathrm{L}$ of the suspensions was taken out and dropped on the coupons. The following fixation was accomplished as described by Allan-Wojtas et al. ${ }^{12}$ The well-prepared specimens were subsequently mounted on aluminum stabs with copper tape, coated with palladium with an ion sputter coater (JEOL JFC1 100; JEOL, Tokyo, Japan). The interaction between the nanoparticles and microorganisms was visualized using FE-SEM (LEO1530; Carl Zeiss Meditec AG).

\section{Inhibition effect of Nano-CHX on I-day biofilms and XTT assay}

As previously described, S. sobrinus and S. mutans were cultured in BHI broth and incubated overnight. Then the bacteria were centrifuged and washed with phosphate-buffered saline (PBS) twice and re-suspended with BHI at a concentration of $10^{9}$ cells $/ \mathrm{mL}$. In all, $200 \mu \mathrm{L}$ of the well-prepared cell suspension was subsequently pipetted in 96-well plates and incubated in the anaerobic chamber. After 24 hours, the bacteria biofilms were washed with PBS to remove loosely attached cells. ${ }^{7}$ C. albicans was cultured to form biofilms as described by Chandra et al. ${ }^{13}$ The overnight broth-cultured C. albicans was centrifuged and washed with PBS twice and then re-suspended in YNB containing $100 \mathrm{mM}$ glucose at a concentration of $10^{7}$ cells $/ \mathrm{mL}$. The cells were then inoculated into the 96-well plates and allowed to attach to the plate bottom at $37^{\circ} \mathrm{C}, 80 \mathrm{rpm}$ for 90 minutes. Then, the biofilms in the early stage were aspirated to remove the non-adherent yeast cells and washed gently with PBS twice and then matured in YNB containing $100 \mathrm{mM}$ glucose. After 24 hours of incubation, both the 1-day-old bacterial and yeast biofilms were treated with $\mathrm{CHX}, \mathrm{S}-/ \mathrm{W}-\mathrm{CHX}$ (containing same drug amount as free $\mathrm{CHX}$ at each concentration), and S-/W-MSNs (blank nanoparticles with same weight as S-/W-CHX) in different concentrations for 24 hours. After the treatment, the biofilms were washed gently with $100 \mu \mathrm{L}$ of PBS, and their vitalities were measured by a 2,3-bis (2-methoxy-4nitro-5-sulfo-phenyl)-2H-tetrazolium-5-carboxanilide (XTT) reduction assay with some modifications. ${ }^{14}$ Then, $200 \mu \mathrm{L}$ of freshly prepared reaction agent (XTT + menadione) was added into each well. After 3 hours of incubation at $37^{\circ} \mathrm{C}$ in the dark, the supernatant of each well was transferred into a new plate and then the plate was assessed at $490 \mathrm{~nm}$ using a microplate reader (SpectraMax M2; Molecular Devices LLC, Sunnyvale, CA, USA). All of the assays were performed on three different occasions in triplicate.

\section{Confocal scanning laser microscopy}

The 1-day-old biofilms were prepared in the chambered cover glass ( $\mu$ Slide 8 well; ibidi GmbH, Munich, Germany), as described previously. Before the treatment, the RITC-S-/ RITC-W-MSNs were dispersed in BHI broth or YNB containing $100 \mathrm{mM}$ glucose by sonication for 10 minutes. Then the RITC-S-/RITC-W-MSNs dispersions were added to the biofilms followed by 24-hour incubation. The treated biofilms were subsequently washed in PBS once to remove the free nanoparticles and then stained with SYTO-9 (Live/ Dead BacLightTM Viability Kit; Thermo Fisher Scientific) for 30 minutes in the dark at room temperature. The images of the fluorescent MSNs in the biofilms were visualized by an Olympus FLUOVIEW FV 1000 confocal scanning laser microscope with FV-10 ASW system (Olympus Corporation, Tokyo, Japan). This microscope equipped with $543 \mathrm{~nm}$ $\mathrm{HeNe}$ laser and $488 \mathrm{~nm}$ Argon laser were used to obtain the red signals from RITC-labeled MSNs and green signals of SYTO-9, respectively. The image analysis was performed with FV10-ASW 4.0 Viewer (Olympus Corporation) and ImageJ. Briefly, the biofilm images with multiple layers were projected over z-axis and transformed into tiff format. The images of red channel, which represents the distribution of RITC-MSNs, were opened with ImageJ to calculate the number of particles, average size, and percentage of RITCMSN area.

\section{Statistical analysis}

The significance of CHX and two different shaped Nano$\mathrm{CHX}$ against the biofilms was analyzed by one-way analysis 
of variance, with multi-comparisons by Tukey's test using GraphPad Prism 6.

\section{Results}

\section{Characterization of S-/W-MSNs, S-/W- $\mathrm{CHX}$ and RITC-S-/RITC-W-MSNs}

The FE-SEM images showed that the diameter of S-MSNs was $265 \pm 30 \mathrm{~nm}$, while the width and length of W-MSNs were $53 \pm 11$ and $477 \pm 152 \mathrm{~nm}$, respectively (Figure 1A and B). The well-ordered pore structures were visualized in transmission electron microscope. The nitrogen adsorption/desorption measurement revealed that S-MSNs had a relatively larger surface area ( 860.52 vs $\left.559.42 \mathrm{~m}^{2} / \mathrm{g}\right)$ but similar pore size (2.86 vs $2.88 \mathrm{~nm}$ ), as compared with W-MSNs (Figure 2).

The loading efficiency of S-CHX $(20.1 \% \pm 0.7 \%)$ was greater than that of W-CHX $(15.8 \% \pm 1.7 \%)$ but without statistical significance. It is worthy to note that their releasing profiles varied. Overall, an initial burst was observed on the two forms of Nano-CHX releasing profiles in the first 8 hours. However, $\mathrm{S}-\mathrm{CHX}$ released more amount of $\mathrm{CHX}$ with reference to
W-CHX after 48 hours (34.0\% vs 22.2\%) (Figure 3). Meanwhile, only a small amount of CHX was released from the nanoparticles within the experimental time, indicating further releasing potential and prolonged effect of nanoparticle encapsulation. Additionally, the emission wavelength of the fluorescent MSNs shifted to $580 \mathrm{~nm}$ with reference to the free RITC (594 nm), due to the conjugation between the RITC and amine group on the particles (Figure 4).

\section{Interactions of MSNs with the planktonic microbes}

As shown in Figure 5, both S- and W-MSNs were found on the microbial surfaces. Notably, SEM displayed clearly that both the shapes of MSNs fused with the microbes tested. Comparably, obvious fusion between the S-MSNs and the microbes existed. The W-MSNs had a larger contact area than the S-MSNs with potentially increased interaction with the microorganisms, and it also enhanced the inter-attachment among the W-MSNs. As such, it may result in a more serious entanglement of W-MSNs than S-MSNs, and affect the
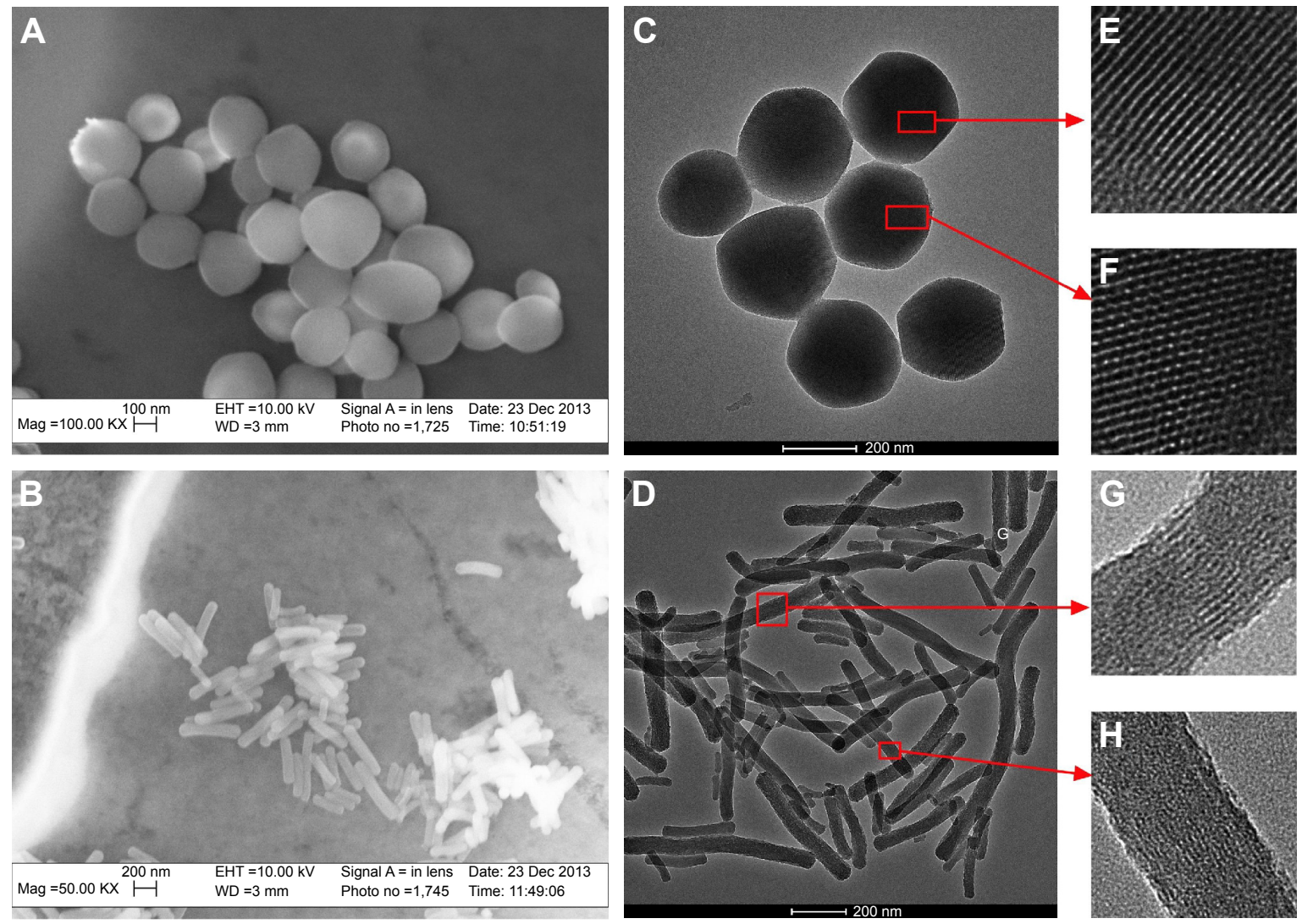

Figure I Characterization of MSN spheres and wires.

Notes: FE-SEM images showing S-MSNs (A) with an average diameter of $265 \pm 30 \mathrm{~nm}$ and W-MSNs (B) with a width and length of $53 \pm \mathrm{II}$ and $477 \pm I 52 \mathrm{~nm}$, respectively. TEM images of S-MSNs (C) and W-MSNs (D) and their pore shapes (E and $\mathbf{F}$; $\mathbf{G}$ and $\mathbf{H}$, respectively).

Abbreviations: Mag, magnification; MSNs, mesoporous silica nanoparticles; FE-SEM, field emission scanning electron microscope; S-MSNs, spherical mesoporous silica nanoparticles; W-MSNs, wire mesoporous silica nanoparticles; TEM, transmission electron microscopy. 
A

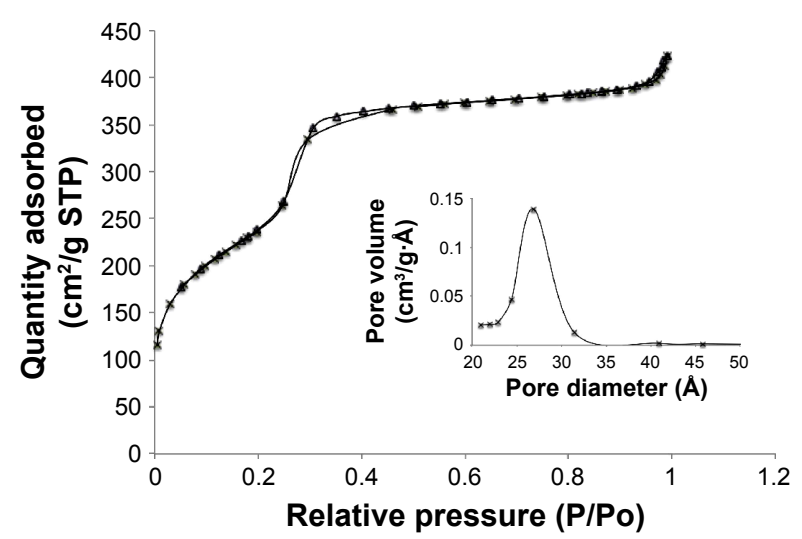

B

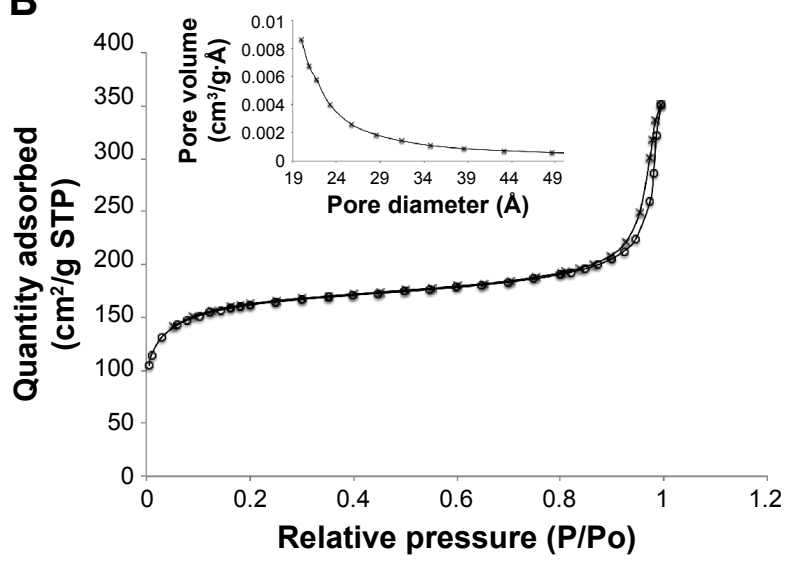

Figure 2 BET nitrogen adsorption-desorption isotherms and $\mathrm{BJH}$ pore size distribution plots of MSN spheres and wires.

Notes: (A) Surface area $\left(860.52 \mathrm{~m}^{2} / \mathrm{g}\right)$ and pore size $\left(2.86 \mathrm{~nm}\right.$; inset) of S-MSNs. (B) Surface area $\left(559.42 \mathrm{~m}^{2} / \mathrm{g}\right)$ and pore size $(2.88 \mathrm{~nm}$; inset) of W-MSNs.

Abbreviations: BET, Brunauer-Emmett-Teller; BJH, Barrett-Joyner-Halenda; MSNs, mesoporous silica nanoparticles; S-MSNs, spherical mesoporous silica nanoparticles; W-MSNs, wire mesoporous silica nanoparticles.

homogeneous dispersion of W-MSNs. Hence, it was found that the entangled W-MSNs could attach to small bacteria ( $<1 \mu \mathrm{m}$ in length) at the end points and relatively larger yeasts $(>2 \mu \mathrm{m}$ in diameter) by the wires.

\section{Anti-biofilm effects of S-CHX and $\mathrm{W}-\mathrm{CHX}$}

The S-/W-MSNs per se did not possess any detectable antibiofilm effects either on bacterial or yeast biofilms (Figure 6). For the monospecies biofilms of $S$. sobrinus, no significant difference was observed between CHX and both S- and $\mathrm{W}-\mathrm{CHX}$ at the concentration of 25 and $100 \mu \mathrm{g} / \mathrm{mL}$. Both the Nano-CHX treated groups showed lower absorbance

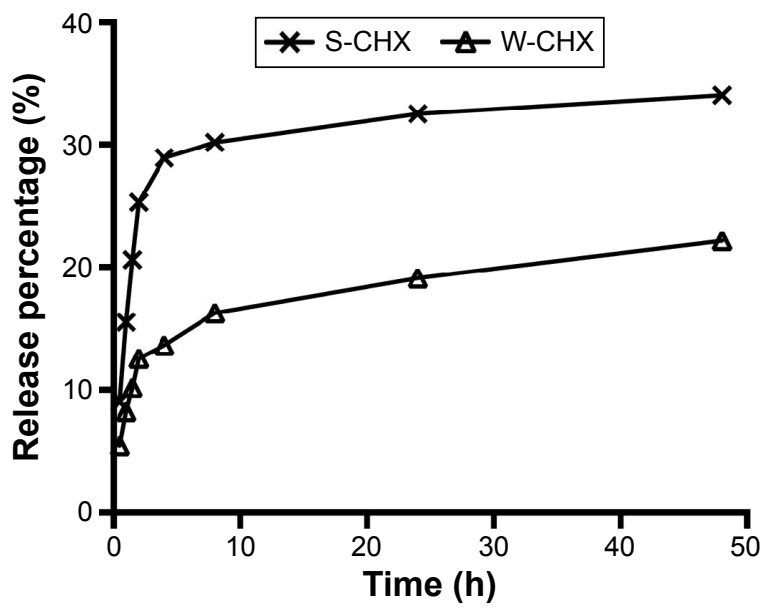

Figure 3 Releasing profile of $\mathrm{CHX}$ from two different shaped nanoparticleencapsulated $\mathrm{CHX}$.

Notes: The release percentages of $\mathrm{CHX}$ from S-CHX and W-CHX in distilled water at each time point were acquired by measuring the absorbance using UV-visible absorption spectroscopy at $254 \mathrm{~nm}$.

Abbreviations: $\mathrm{CHX}$, chlorhexidine; S-CHX, spherical nanoparticle-encapsulated chlorhexidine; W-CHX, wire nanoparticle-encapsulated chlorhexidine. compared to $\mathrm{CHX}(50 \mu \mathrm{g} / \mathrm{mL})$ alone. For the biofilms of S. mutans, S-CHX exhibited the strongest inhibitory effect at $3.125 \mu \mathrm{g} / \mathrm{mL}$. Interestingly, it exhibited potent anti-biofilm effects on C.albicans biofilms with reference to other groups of treatment at all tested concentrations.

\section{Diffusion of fluorescent MSNs in microbial biofilms}

The interactions of nanoparticles with the microbial biofilms were further investigated by confocal microscopy (Figure 7). Fluorescent MSNs presented with red dots could be observed in monospecies biofilms of $S$. sobrinus, S. mutans, and C. albicans. The penetration and distribution of RITC-MSNs

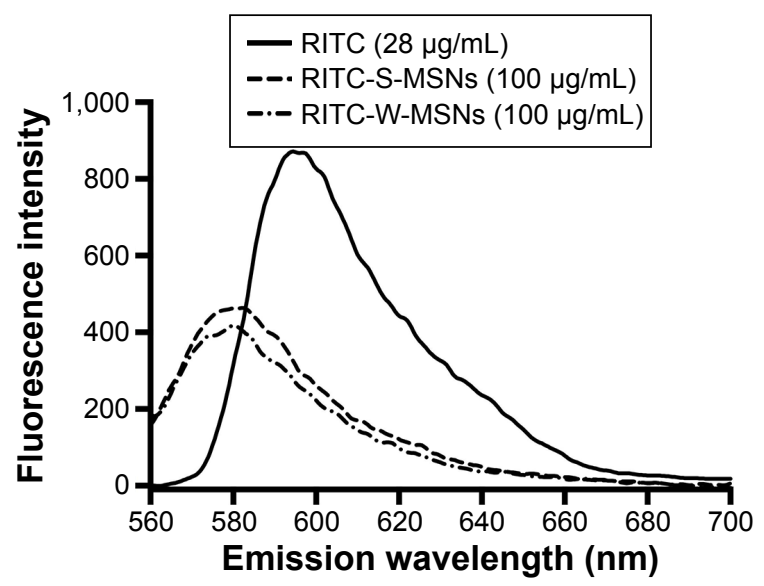

Figure 4 Fluorescence emission spectra of fluorescent MSN spheres and wires. Notes: After being excited at $543 \mathrm{~nm}$, the emission wavelength of RITC-S-MSNs and RITC-W-MSNs (580 nm) showed a blue sift compared with RITC (594 nm). Abbreviations: MSNs, mesoporous silica nanoparticles; RITC, rhodamine B isothiocyanate; RITC-S-MSNs, rhodamine B isothiocyanate-labeled spherical mesoporous silica nanoparticles; RITC-W-MSNs, rhodamine B isothiocyanatelabeled wire mesoporous silica nanoparticles. 

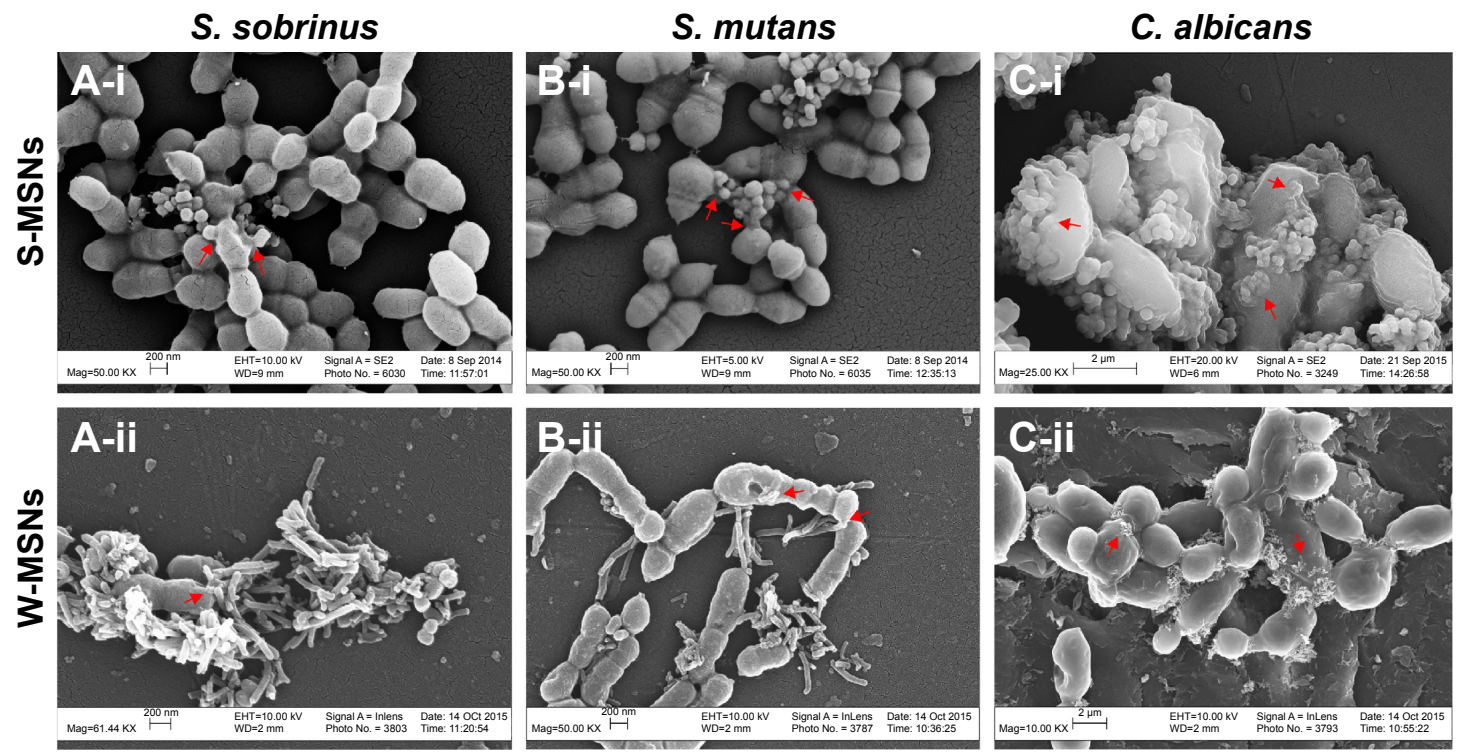

Figure 5 FE-SEM images of the interactions of S-MSNs or W-MSNs with planktonic microorganisms.

Note: S. sobrinus (A-i and ii), S. mutans (B-i and ii), and C. albicans (C-i and ii). The arrows highlight the interactive spots of MSNs with microbes.

Abbreviations: FE-SEM, field emission scanning electron microscope; S-MSNs, spherical mesoporous silica nanoparticles; W-MSNs, wire mesoporous silica nanoparticles; S. sobrinus, Streptococcus sobrinus; S. mutans, Streptococcus mutans; C. albicans, Candida albicans; Mag, magnification; EHT, extra high tension; WD, working distance; MSNs, mesoporous silica nanoparticles.

were quantitatively analyzed using ImageJ (Table 1). Interestingly, more spherical fluorescent MSNs were detected in the three forms of biofilms, while the RITC-W-MSNs aggregated greatly to form larger particles in the monospecies biofilms. Moreover, the RITC-S-MSNs dispersed and distributed fairly evenly with larger detectable diffusion area with reference to the RITC-W-MSNs.

\section{Discussion}

The particle's morphology, size, and pore volume are the critical factors to determine the surface area of the MSNs, which subsequently affect the drug loading efficiency. ${ }^{15}$ It is widely accepted that larger surface area and pore volume of nanoparticles provide more space for drug accommodation. In the present study, we found that S-CHX, which has a larger surface area, showed a tendency of higher loading efficiency than W-CHX, although the difference did not reach statistical significance. Next, both the shapes of nanoparticle encapsulation per se could extend $\mathrm{CHX}$ releasing up to 48 hours. Notably, S-CHX showed a faster releasing rate than W-CHX in the time-course experiment. It could be due to the relatively large surface area of S-MSNs that increased contact area of the working solution with the nanoparticles, and subsequently promoted CHX diffusion from the pores of particles. ${ }^{16}$ Moreover, the entanglement was more commonly observed in W-MSNs than in S-MSNs since the former provided a relatively large contact area than the latter, which further decreased the surface area of W-MSNs and thereby may result in a slower $\mathrm{CHX}$ releasing profile.

The entanglement was further confirmed in the interactions of MSNs with microorganisms. More agglomerated W-MSNs were found than S-MSNs under FE-SEM, although the MSNs had been sonicated for 10 minutes before adding to the microbial suspensions. After co-incubation for 24 hours, some MSNs were found to attach on the microbes. Moreover, the S-MSNs could be fused with the surfaces of microbes more obviously than the W-MSNs. Previous studies have examined the interactive profiles of nanoparticles with bacteria and explored the underlying mechanisms. The polyurethane nanoparticles generated from the prostheses were found on the cell wall of Staphylococcus aureus and in its cytoplasm. ${ }^{17}$ Besides the polyurethane nanoparticles, gold nanoparticles coated by citrate or bovine serum albumin are associated with the surface as well as the intracellular cytoplasm of Escherichia coli. ${ }^{18}$ It has also been well documented that the nanoparticles (quantum dots and metal oxides) could also be internalized by the bacteria other than by endocytosis. ${ }^{19,20}$ The quantum dots with mean diameter less than $6 \mathrm{~nm}$ can diffuse into bacteria through membrane pores. ${ }^{19}$ The metal nanoparticles with a large size could diffuse into the cells through the damage to cell membrane. ${ }^{21}$ Moreover, the conjugation of nanoparticles with different molecules may increase the internalization of large size nanoparticles. ${ }^{18}$ In the present study, the 

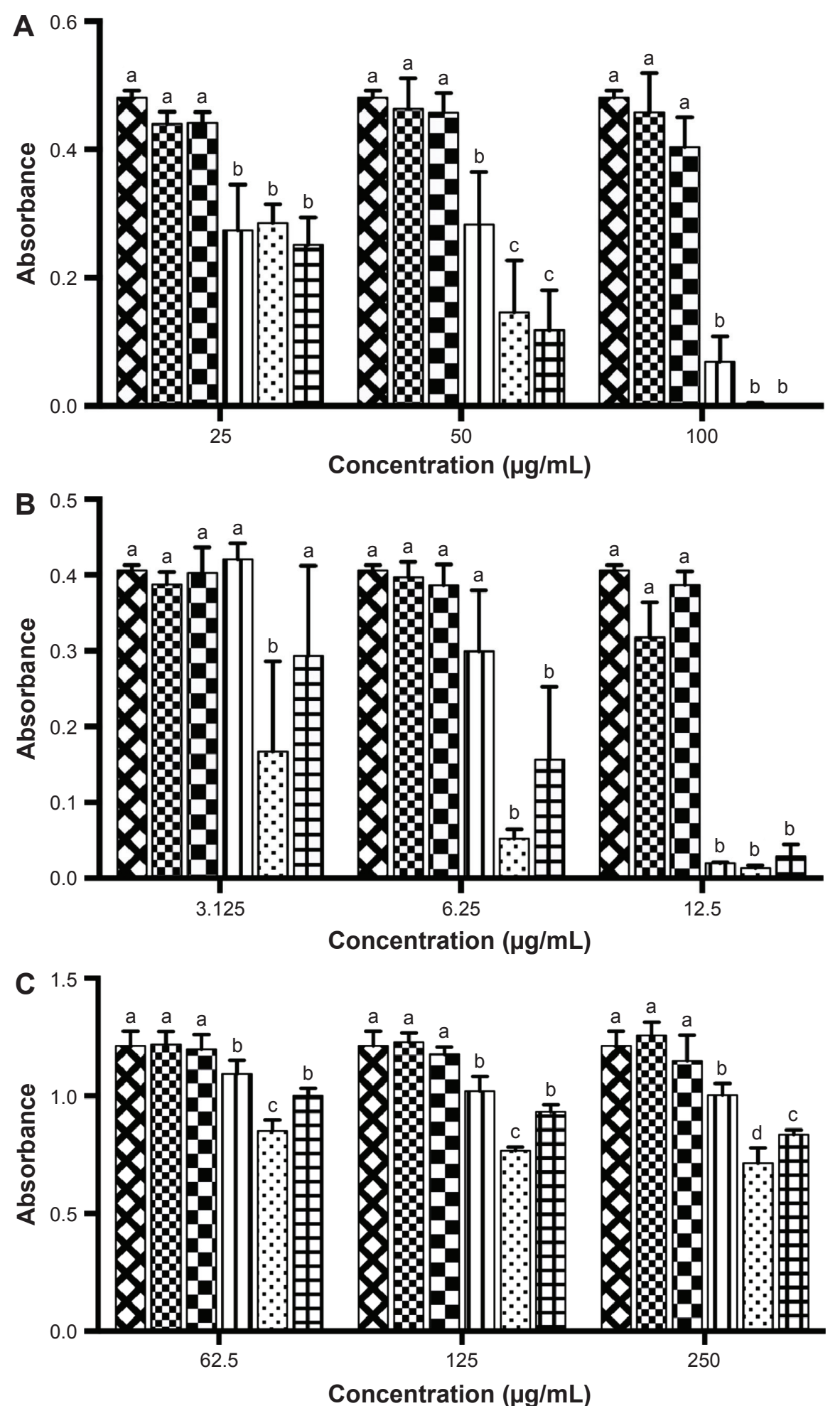

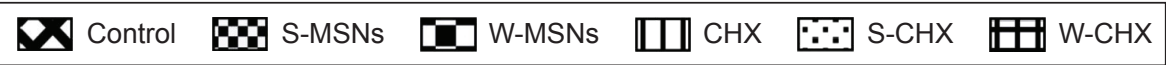

Figure 6 Absorbance (490 nm) of I-day-old microorganism biofilms after being treated with free and two different shaped nanoparticle-encapsulated CHX (XTT assay). Notes: (A) S. sobrinus, (B) S. mutans, and (C) C. albicans. S- and W-CHX encapsulated with the same drug amount as the free CHX. S- and W-MSNs were equivalent to $\mathrm{S}$ - and $\mathrm{W}$ - $\mathrm{CHX}$ at each concentration. The values on the $\mathrm{x}$-axes refer to the concentrations of free or nanoparticle-encapsulated $\mathrm{CHX}$. The lowercase letters above each bar (a, b, c, and d) indicate significant differences $(P<0.05)$ between the control and treatment groups.

Abbreviations: $\mathrm{CHX}$, chlorhexidine; XTT, 2,3-bis (2-methoxy-4-nitro-5-sulfo-phenyl)-2H-tetrazolium-5-carboxanilide; S. sobrinus, Streptococcus sobrinus; S. mutans, Streptococcus mutans; C. albicans, Candida albicans; S-CHX, spherical nanoparticle-encapsulated chlorhexidine; W-CHX, wire nanoparticle-encapsulated chlorhexidine; S-MSNs, spherical mesoporous silica nanoparticles; W-MSNs, wire mesoporous silica nanoparticles. 

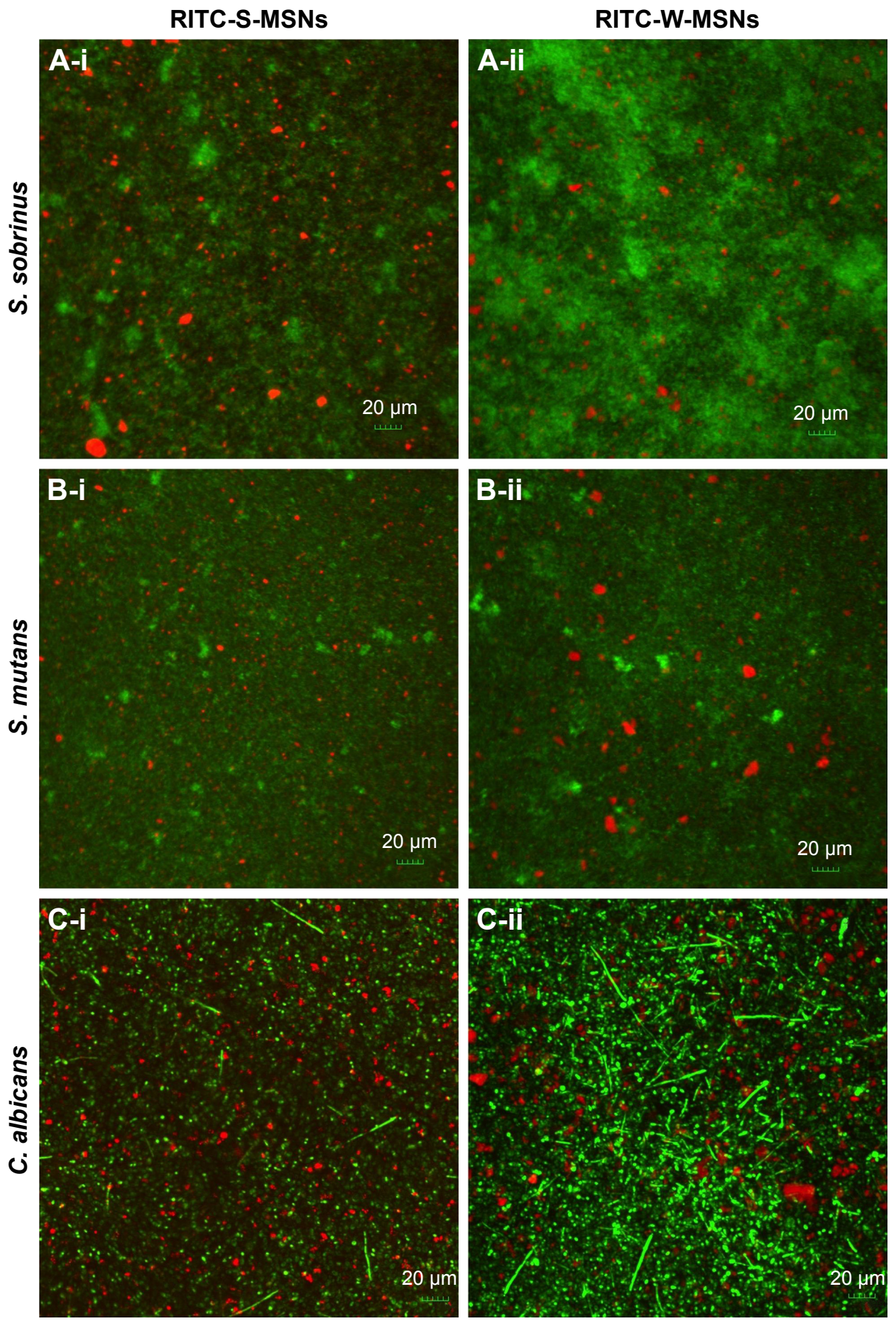

Figure 7 Confocal images of RITC-S-MSNs and RITC-W-MSNs (50 $\mu \mathrm{g} / \mathrm{mL}$ for bacteria and $100 \mu g / \mathrm{mL}$ for yeast) in I-day-old microorganism biofilms after 24 hours of incubation.

Note: S. sobrinus (A-i and ii), S. mutans (B-i and ii), and C. albicans (C-i and ii).

Abbreviations: RITC-S-MSNs, rhodamine B isothiocyanate-labeled spherical mesoporous silica nanoparticles; RITC-W-MSNs, rhodamine B isothiocyanate-labeled wire mesoporous silica nanoparticles; S. sobrinus, Streptococcus sobrinus; S. mutans, Streptococcus mutans; C. albicans, Candida albicans.

blank MSN particles without any modification were used to investigate their interactions with cells. These blank nanoparticles per se have no antibacterial effects without damaging cellular membrane. ${ }^{7,22}$ Therefore, it is conceivable that the blank MSNs with a relatively large size (more than $100 \mathrm{~nm}$ ) could not diffuse into the cytoplasm by causing damage to the cell walls. In this study, the images captured by FE-SEM confirm the adhesion and fusion of the MSNs with the microorganisms, likely due to the biocompatibility between their hydroxyl groups and cell walls. 
Table I The number of nanoparticles, average size, and percentage of two different shaped RITC-MSNs areas in the monospecies biofilms of S. sobrinus, S. mutans, and C. albicans

\begin{tabular}{lllll}
\hline $\begin{array}{l}\text { Microbel } \\
\text { nanoparticles }\end{array}$ & $\begin{array}{l}\text { Number of } \\
\text { nanoparticles }\end{array}$ & $\begin{array}{l}\text { Average } \\
\text { size }(\mu \mathrm{m})\end{array}$ & $\begin{array}{l}\text { Area } \\
\left(\mu \mathrm{m}^{2}\right)\end{array}$ & Area\% \\
\hline $\begin{array}{l}\text { S. sobrinus } \\
\text { RITC-S-MSNs }\end{array}$ & 2,031 & 3.5 & $7,259.3$ & 7.2 \\
RITC-W-MSNs & 736 & 6.3 & $4,631.4$ & 4.7 \\
S. mutans & & & & \\
RITC-S-MSNs & 2,101 & 2.6 & $5,477.0$ & 5.5 \\
RITC-W-MSNs & 579 & 6.6 & $3,822.1$ & 3.9 \\
C. albicans & & & & \\
RITC-S-MSNs & 1,894 & 4.5 & $8,519.4$ & 8.6 \\
RITC-W-MSNs & I,0I4 & 8.0 & $8,089.5$ & 8.2 \\
\hline
\end{tabular}

Notes: The number of nanoparticles, average size, and area\% were calculated on the basis of the red channel of the confocal images. The number of nanoparticles represents the number of countable particles with fixed hue, saturation, and brightness.

Abbreviations: RITC-MSNs, rhodamine B isothiocyanate-labeled mesoporous silica nanoparticles; RITC-S-MSNs, rhodamine B isothiocyanate-labeled spherical mesoporous silica nanoparticles; RITC-W-MSNs, rhodamine B isothiocyanatelabeled wire mesoporous silica nanoparticles; S. sobrinus, Streptococcus sobrinus; S. mutans, Streptococcus mutans; C. albicans, Candida albicans.

Both bacteria and yeasts can produce biopolymer matrix which contributes to form and develop microbial biofilms, thereby enhancing the antibiotic resistance of microorganism. ${ }^{23,24}$ Several hypotheses have been proposed to explain the biofilm resistance to antibiotics, such as drug exclusion by the biopolymer matrix and formation of persisters. ${ }^{25}$ In order to tackle antibiotic resistance, lipid and polymer nanoparticles have been widely used as promising drug vehicles for delivering antibiotics into the biofilms. ${ }^{26} \mathrm{In}$ addition, metal or metal oxide nanoparticles, such as silver, gold, iron oxide, and zinc oxide, have been developed to effectively control microbial biofilms through penetrating biopolymer matrix and causing damage to the cell walls. ${ }^{27-29}$ Apart from the materials already mentioned, silica nanoparticles with nitric oxide donators also exhibit potent antibiofilm efficiency. ${ }^{6}$ Moreover, Slomberg et al found that the decreased size and increased aspect ratio could enhance the anti-biofilm efficiency of the NO-releasing silica nanoparticles. ${ }^{5}$ However, in our study, W-CHX with wire shape did not show a better anti-biofilm efficiency than S-CHX, partly due to the entanglement of W-CHX that may restrict the release of CHX. The nanoparticle encapsulation per se still improves the anti-biofilm efficiency of CHX. It could be attributed to the protective effect of the nanoparticles on this anti-biofilm agent during the penetration of the biopolymer matrix. Additionally, Nano-CHX, especially S-CHX, appears to interact closely with microorganisms and releases the drug directly within the biofilms after the penetration. In this study, the penetration of Nano-CHX was further investigated indirectly by the fluorescent MSNs. Interestingly, these fluorescent MSNs can be found within the biofilms, while RITC-SMSNs could form smaller agglomerates and distribute more evenly than RITC-W-MSNs. Currently, many researchers have addressed the general steps of the interactions between the nanoparticles and biofilms, and how the surface modifications could affect the penetration of particles into the biofilms. ${ }^{30}$ Although fluorescent MSNs and Nano-CHX have the same core structures, they remain to vary in the surface functional groups. This might result in the different interactions with the biofilms. Hence, the development of hybrid nanoparticles with fluorescent groups on the surface and inside-encapsulated antibiotics is highly warranted for further studies on the pathways and performance of nanoparticles in their interactions with microbial biofilms.

In summary, the S-MSNs had a larger surface area and released encapsulated CHX faster than the W-MSNs. The SEM images showed that the S-MSNs could fuse and have closer interactions with the oral microorganisms than the W-MSNs. Both S-MSNs and W-MSNs could enhance the anti-biofilm efficiencies of CHX through effective nanoparticle encapsulation. Moreover, the S-CHX exhibited a higher anti-biofilm activity than the W-CHX, since the entanglement of W-CHX could restrict the drug release and interactions with the microorganisms. The confocal images confirmed that the fluorescent MSNs were able to penetrate the biofilms, and RITC-S-MSNs showed relatively more homogeneous distribution than the RITC-W-MSNs. In conclusion, the present study reveals that S-CHX may preferably enhance its anti-biofilm efficiency through an effective releasing mode and close interactions with microbes.

\section{Acknowledgments}

This work was supported by the General Research Fund from Hong Kong Research Grants Council (HKU767512M and HKBU201213). We thank Professor Shouhu Xuan from University of Science and Technology of China for the measurements of nanoparticle surface areas and pore sizes, and Dr Sarah SW Wong from The University of Hong Kong for technical support.

\section{Disclosure}

The authors report no conflicts of interest in this work.

\section{References}

1. Tao Z. Mesoporous silica-based nanodevices for biological applications RSC Adv. 2014;4:18961-18980.

2. Meng H, Xue M, Xia T, et al. Autonomous in vitro anticancer drug release from mesoporous silica nanoparticles by $\mathrm{pH}$-sensitive nanovalves. $\mathrm{J} \mathrm{Am}$ Chem Soc. 2010;132(36):12690-12697. 
3. Lu J, Liong M, Li Z, Zink JI, Tamanoi F. Biocompatibility, biodistribution, and drug-delivery efficiency of mesoporous silica nanoparticles for cancer therapy in animals. Small. 2010;6(16):1794-1805.

4. Lee $\mathrm{CH}$, Cheng SH, Huang I, et al. Intracellular $\mathrm{pH}$-responsive mesoporous silica nanoparticles for the controlled release of anticancer chemotherapeutics. Angew Chem Int Edit Engl. 2010;122(44):8390-8395.

5. Slomberg DL, Lu Y, Broadnax AD, Hunter RA, Carpenter AW, Schoenfisch MH. Role of size and shape on biofilm eradication for nitric oxide-releasing silica nanoparticles. ACS Appl Mater Interfaces. 2013; 5(19):9322-9329.

6. Hetrick EM, Shin JH, Paul HS, Schoenfisch MH. Anti-biofilm efficacy of nitric oxide-releasing silica nanoparticles. Biomaterials. 2009;30(14): 2782-2789.

7. Seneviratne CJ, Leung KC, Wong CH, et al. Nanoparticle-encapsulated chlorhexidine against oral bacterial biofilms. PLoS One. 2014;9(8): e103234.

8. Asefa T, Tao Z. Biocompatibility of mesoporous silica nanoparticles. Chem Res Toxicol. 2012;25(11):2265-2284.

9. Huang X, Teng X, Chen D, Tang F, He J. The effect of the shape of mesoporous silica nanoparticles on cellular uptake and cell function. Biomaterials. 2010;31(3):438-448.

10. Yu T, Malugin A, Ghandehari H. Impact of silica nanoparticle design on cellular toxicity and hemolytic activity. ACS Nano. 2011;5(7): 5717-5728

11. Yamaguchi A, Namekawa M, Itoh T, Teramae N. Microviscosity of supercooled water confined within aminopropyl-modified mesoporous silica as studied by time-resolved fluorescence spectroscopy. Anal Sci. 2012;28(11):1065-1070.

12. Allan-Wojtas P, Hansen LT, Paulson A. Microstructural studies of probiotic bacteria-loaded alginate microcapsules using standard electron microscopy techniques and anhydrous fixation. LWT Food Sci Technol. 2008;41(1):101-108.

13. Chandra J, Mukherjee PK, Ghannoum MA. In vitro growth and analysis of Candida biofilms. Nat Protoc. 2008;3(12):1909-1924.

14. Li MY, Huang RJ, Zhou XD, Gregory RL. Role of sortase in Streptococcus mutans under the effect of nicotine. Int J Oral Sci. 2013;5(4): 206-211.

15. Liu Y, Tan J, Thomas A, Ou-Yang D, Muzykantov VR. The shape of things to come: importance of design in nanotechnology for drug delivery. Ther Deliv. 2012;3(2):181-194.

16. Liu Y, Sun C, Hao Y, Jiang T, Zheng L, Wang S. Mechanism of dissolution enhancement and bioavailability of poorly water soluble celecoxib by preparing stable amorphous nanoparticles. J Pharm Pharm Sci. 2010;13(4):589-606.
17. Curia R, Milani M, Didenko LV, Avtandilov GA, Shevlyagina NV, Smirnova TA. Beyond the Biodestruction of Polyurethane: S. aureus Uptake of Nanoparticles is a Challenge for Toxicology. Vol 1. Badajoz: Formatex Research Center; 2014.

18. McQuillan J. Bacterial-Nanoparticle Interactions [doctoral thesis]. Exeter: University of Exeter; 2010.

19. Kloepfer JA, Mielke RE, Nadeau JL. Uptake of CdSe and CdSe/ZnS quantum dots into bacteria via purine-dependent mechanisms. Appl Environ Microbiol. 2005;71(5):2548-2557.

20. Kumar A, Pandey AK, Singh SS, Shanker R, Dhawan A. A flow cytometric method to assess nanoparticle uptake in bacteria. Cytometry A. 2011; 79(9):707-712.

21. Yan D, Yin G, Huang Z, et al. Cellular compatibility of biomineralized $\mathrm{ZnO}$ nanoparticles based on prokaryotic and eukaryotic systems. Langmuir. 2011;27(21):13206-13211.

22. Trewyn BG, Whitman CM, Lin VSY. Morphological control of room-temperature ionic liquid templated mesoporous silica nanoparticles for controlled release of antibacterial agents. Nano Lett. 2004;4(11):2139-2143.

23. Chandra J, Kuhn DM, Mukherjee PK, Hoyer LL, McCormick T, Ghannoum MA. Biofilm formation by the fungal pathogen Candida albicans: development, architecture, and drug resistance. J Bacteriol. 2001;183(18):5385-5394

24. Høiby N, Bjarnsholt T, Givskov M, Molin S, Ciofu O. Antibiotic resistance of bacterial biofilms. Int J Antimicrob Agents. 2010;35(4): 322-332.

25. Stewart PS. Mechanisms of antibiotic resistance in bacterial biofilms. Int J Med Microbiol. 2002;292(2):107-113.

26. Forier K, Raemdonck K, De Smedt SC, Demeester J, Coenye T, Braeckmans K. Lipid and polymer nanoparticles for drug delivery to bacterial biofilms. J Control Release. 2014;190:607-623.

27. Lee JH, Kim YG, Cho MH, Lee J. ZnO nanoparticles inhibit Pseudomonas aeruginosa biofilm formation and virulence factor production. Microbiol Res. 2014;169(12):888-896.

28. Pérez-Díaz MA, Boegli L, James G, et al. Silver nanoparticles with antimicrobial activities against Streptococcus mutans and their cytotoxic effect. Mater Sci Eng C Mater Biol Appl. 2015;55:360-366.

29. Sathyanarayanan MB, Balachandranath R, Genji Srinivasulu Y, Kannaiyan SK, Subbiahdoss G. The effect of gold and iron-oxide nanoparticles on biofilm-forming pathogens. ISRN Microbiol. 2013; 2013:272086.

30. Ikuma K, Decho AW, Lau BL. When nanoparticles meet biofilmsInteractions guiding the environmental fate and accumulation of nanoparticles. Front Microbiol. 2015;6:591.
International Journal of Nanomedicine

\section{Publish your work in this journal}

The International Journal of Nanomedicine is an international, peerreviewed journal focusing on the application of nanotechnology in diagnostics, therapeutics, and drug delivery systems throughout the biomedical field. This journal is indexed on PubMed Central, MedLine, CAS, SciSearch ${ }^{\circledR}$, Current Contents ${ }^{\circledR} /$ Clinical Medicine,
Dovepress

Journal Citation Reports/Science Edition, EMBase, Scopus and the Elsevier Bibliographic databases. The manuscript management system is completely online and includes a very quick and fair peer-review system, which is all easy to use. Visit http://www.dovepress.com/ testimonials.php to read real quotes from published authors. 